\title{
Latent Class Growth Modelling: A Tutorial
}

\author{
Heather Andruff, Natasha Carraro, Amanda Thompson, and Patrick Gaudreau \\ University of Ottawa \\ Benoît Louvet \\ Université de Rouen
}

\begin{abstract}
The present work is an introduction to Latent Class Growth Modelling (LCGM). LCGM is a semi-parametric statistical technique used to analyze longitudinal data. It is used when the data follows a pattern of change in which both the strength and the direction of the relationship between the independent and dependent variables differ across cases. The analysis identifies distinct subgroups of individuals following a distinct pattern of change over age or time on a variable of interest. The aim of the present tutorial is to introduce readers to LCGM and provide a concrete example of how the analysis can be performed using a real-world data set and the SAS software package with accompanying PROC TRAJ application. The advantages and limitations of this technique are also discussed.
\end{abstract}

Longitudinal data is at the core of research exploring change in various outcomes across a wide range of disciplines. A number of statistical techniques are available for analyzing longitudinal data (see Singer \& Willet, 2003).

Heather Andruff, Natasha Carraro, Amanda Thompson, and Patrick Gaudreau, School of Psychology, University of Ottawa; Benoît Louvet, Départment du Sport et l'Éducation Physique, Université de Rouen. Heather Andruff, Natasha Carraro, and Amanda Thompson played an equal role in the preparation of this manuscript and should all be considered as first authors. This research was supported by a Social Sciences and Humanities Research Council Canada Graduate Scholarship awarded to Natasha Carraro, an Ontario Graduate Scholarship awarded to Amanda Thompson, and a regular research grant from the Social Sciences and Humanities Research Council and the University of Ottawa Research Scholar Fund awarded to Patrick Gaudreau.

Correspondence concerning this article should be sent to Heather Andruff, University of Ottawa, School of Psychology, 145 Jean-Jacques Lussier St., Ottawa, Ontario, Canada, K1N 6N5. Email: heather.andruff@uottawa.ca.
One approach is to study raw change scores. By this method, change is computed as the difference between the Time 1 and the Time 2 scores and the resulting raw change values are analyzed as a function of individual or group characteristics (Curran \& Muthén, 1999). Raw change scores are typically analyzed using $t$-tests, analysis of variance (ANOVA), or multiple regression. An alternative approach is to study residualized change scores. By this method, change is computed as the residual between the observed Time 2 score and the expected Time 2 score as predicted by the Time 1 score (Curran \& Muthén, 1999). Residualized change scores are typically analyzed using multiple regression or analysis of covariance (ANCOVA).

Although both raw and residualized change scores can be useful for analyzing longitudinal data under some circumstances, one limitation is that they tend to consider change between only two discrete time points and are thus more useful for prospective research designs (Curran \& Muthén, 1999). Frequently, however, researchers are interested in modelling developmental trajectories, or patterns of change in an outcome across multiple (i.e., at least three) time points (Nagin, 2005). For instance, psychologists try to identify the course of various psychopathologies (e.g., Maughan, 2005), criminologists study the progression of 
criminality over life stages (e.g., Sampson \& Laub, 2005), and medical researchers test the impact of various treatments on the progression of disease (e.g., Llabre, Spitzer, Siegel, Saab, \& Schneiderman, 2004).

A common approach to studying developmental trajectories is to use standard growth analyses such as repeated measures multivariate analysis of variance (MANOVA) or structural equation modelling (SEM; Jung \& Wickrama, 2008). Standard growth analyses estimate a single trajectory that averages the individual trajectories of all participants in a given sample. Time or age is used as an independent variable to delineate the strength and direction of an average pattern of change (i.e., linear, quadratic, or cubic) across time for an entire sample. This average trajectory contains an averaged intercept (i.e., the expected value of the dependent variable when the value of the independent variable(s) is/are equal to zero) and an averaged slope (i.e., a line representing the predicted strength and direction of the growth trend) for the entire sample. This approach captures individual differences by estimating a random coefficient that represents the variability surrounding this intercept and slope. By this method, researchers can use categorical or continuous independent variables, representing potential risk or protective factors, to predict individual differences in the intercept and/or slope values. By centering the age or time variable, a researcher may set the intercept to any predetermined value of interest. For instance, a researcher could use self-esteem to predict individual differences in the intercept of depression at the start, middle, or end of a semester, depending on the research question. Results could indicate that people with higher self-esteem report lower levels of depression at the start of the semester. Similarly, researchers could use self-esteem to predict individual differences in the linear slope of depression. Results could indicate that people with higher self-esteem at baseline experience a slower increase in depressive symptoms over the course of the semester, indicating that self-esteem is a possible protective factor against a more severe linear increase in depression.

Standard growth models are useful for studying research questions for which all individuals in a given sample are expected to change in the same direction across time with only the degree of change varying between people (Raudenbush, 2001). Nagin (2002) offers time spent with peers as an example of this monotonic heterogeneity of change. With few exceptions, children tend to spend more time with their peers as they move from childhood to adolescence. In this case, it is useful to frame a research question in terms of an average trajectory of time spent with peers. However, some psychological phenomena may follow a multinomial pattern in which both the strength and the direction of change are varying between people (Nagin, 2002). Raudenbush (2001) uses depression as an example by arguing that it is incorrect to assume that all people in a given sample would be experiencing either increasing or decreasing levels of depression. In a normative sample, he states, many people will never be high on depression, others will always be high, others will become increasingly depressed, while others may fluctuate between high and low levels of depression. In such instances, a single averaged growth trajectory could mask important individual differences and lead to the erroneous conclusion that people are not changing on a given variable. Such conclusions could be drawn if $50 \%$ of the sample increased by the same amount on a particular variable whereas $50 \%$ of the sample decreased by the same amount on that variable. Here, a single growth trajectory would average to zero, thus prompting researchers to conclude an absence of change despite the presence of substantial yet opposing patterns of change for two distinct subgroups in the sample (Roberts, Walton, \& Viechtbauer, 2006). For this class of problems, alternative modelling strategies are available that consider multinomial heterogeneity in change. One such approach is a group-based statistical technique known as Latent Class Growth Modelling (LCGM).

\section{Theoretical basis of LCGM}

Given the substantial contribution of Nagin (1999; 2005) to both the theory and methodology of LCGM, the following explanations of the technique draw primarily from his work and from recent extensions proposed by his collaborators. LCGM is a semi-parametric technique used to identify distinct subgroups of individuals following a similar pattern of change over time on a given variable. Although each individual has a unique developmental course, the heterogeneity or the distribution of individual differences in change within the data is summarized by a finite set of unique polynomial functions each corresponding to a discrete trajectory (Nagin, 2005). Given that the magnitude and direction of change can vary freely across trajectories, a set of model parameters (i.e., intercept and slope) is estimated for each trajectory (e.g., Nagin, 2005). Unlike standard latent growth modelling techniques in which individual differences in both the slope and intercept are estimated using random coefficients, LCGM fixes the slope and the intercept to equality across individuals within a trajectory. Such an approach is acceptable given that individual differences are captured by the multiple trajectories included in the model. Given that both the slope and intercept are fixed, a degree of freedom remains available to estimate quadratic trajectories of a variable measured at three time points or cubic trajectories with data 
available at four time points.

Although the model is widely applicable, the rating scale of the instrument used to measure the variable of interest dictates the specific probability distribution used to estimate the parameters. Psychometric scale data necessitate the use of the censored normal model distribution, dichotomous data require the use of the binary logit distribution, and frequency data dictate the use of the Poisson distribution. For example, in the censored normal model, each trajectory is described as a latent variable $\left(y_{i t}^{*}\right)$ that represents the predicted score on a given dependent variable of interest $(\mathrm{Y})$ for a given trajectory $(j)$ at a specific time $(t)$ and is defined by the following function:

$$
\left(y_{i t}^{*}\right)=\beta_{0}^{j}+\beta_{1}^{j} \mathrm{X}_{i t}+\beta_{2}^{j} \mathrm{X}_{i t}^{2}+\beta_{3}^{j} \mathrm{X}_{i t}^{3}+\varepsilon_{i t}
$$

In this equation, $\mathrm{X}_{i t}, \mathrm{X}_{i t}^{2}$, and $\mathrm{X}_{i t}^{3}$ represent the independent variable (i.e., Time or Age) entered in a regular, squared, or cubed term, respectively. Further, $\varepsilon_{i t}$ is a disturbance term assumed to be normally distributed with a mean of zero and a constant standard deviation. Finally, $\beta_{0}^{j}, \beta_{1}^{j}, \beta_{2}^{j}$, and $\beta_{3}^{j}$ are the parameters defining the intercept and slopes (i.e., linear, quadratic, cubic) of the trajectory for a specific subgroup $(j)$. As demonstrated in the above polynomial function, the trajectories are most often modelled using either a linear $\left(\mathrm{X}_{i t}\right)$, quadratic $\left(\mathrm{X}_{i t}^{2}\right)$, or cubic $\left(\mathrm{X}_{i t}^{3}\right)$ trend, depending on the number of time points measured. A linear pattern of change is defined by the $\left(\mathrm{X}_{i t}\right)$ parameter and a linear trend may either steadily increase or decrease at varying magnitudes or remain stable. A quadratic pattern of change is defined by the $\left(\mathrm{X}_{i t}^{2}\right)$ parameter and a quadratic trend may increase, decrease, or remain stable up to a certain time point before changing in either magnitude or direction. Furthermore, a cubic trajectory is defined by the $\left(\mathrm{X}_{i t}^{3}\right)$ parameter and a cubic trend will have two changes in either the magnitude or direction across time points.

Using LCGM, researchers must specify the number of distinct trajectories to be extracted from the data and select the model with the number of trajectories that best fits the data. It is preferable to have a priori knowledge concerning the number and the shape of trajectories whenever theory and literature exists in the area of study. Researchers evaluate which model provides the best fit to the data by interpreting and comparing both the fit statistics and the posterior probabilities for each model tested. The Bayesian Information Criterion (BIC) value is obtained for each model tested and is a fit index used to compare competing models that include different numbers of trajectories or trajectories of various shapes (e.g., linear versus quadratic). More specifically, nested models testing the inclusion of a different number of trajectories can be compared using an estimate of the log Bayes Factor defined by the following formula (Jones, Nagin, \& Roeder, 2001):

$$
2 \log _{e}\left(\mathrm{~B}_{10}\right) \approx 2(\mathrm{BIC})
$$

The estimate is approximately equal to two times the difference in the BIC values for the two models being compared. Here, the difference is calculated by subtracting the BIC value of the simpler model (i.e., the model with the smaller number of trajectories) from the more complex model (i.e., the model with the larger number of trajectories). A set of guidelines has been adopted for interpreting the estimate of the log Bayes Factor in order to measure the extent of evidence surrounding the more complex model thereby ensuring model parsimony. According to these guidelines, values ranging from 0 to 2 are interpreted as weak evidence for the more complex model, values ranging from 2 to 6 are interpreted as moderate evidence, values ranging from 6 to 10 are interpreted as strong evidence, and values greater than 10 are interpreted as very strong evidence (Jones et al., 2001). Initially, for each model, the linear, quadratic, and cubic functions of each trajectory can be tested, depending on the number of time points. To ensure parsimony, consistent with the recommendations of Helgeson, Snyder, and Seltman (2004), non-significant cubic and quadratic terms are removed from trajectories in a given model, but linear parameters are retained irrespective of significance (as cited in Louvet, Gaudreau, Menaut, Genty, \& Deneuve, 2009). Once nonsignificant terms have been removed, each model is retested yielding a new BIC value. The fit of each nested model is then compared using the estimate of the log Bayes factor. This process of comparing the fit of each subsequent, more complex model, to the fit of the previously tested, simpler model, continues until there is no substantial evidence for improvement in model fit. In addition, both the posterior probabilities and the averaged group membership probabilities for each trajectory are examined to evaluate the tenability of each model.

The parameter coefficients estimated in LCGM provide direct information regarding group membership probabilities. A group membership probability is calculated for each trajectory and corresponds to the aggregate size of each trajectory or the number of participants belonging to a given trajectory. Ideally, each trajectory should hold an approximate group membership probability of at least five percent. However, in clinical samples, some trajectories may model the profile of change of only a fraction of the sample.

Posterior probabilities can be calculated post hoc to estimate the probability that each case, with its associated profile of change, is a member of each modelled trajectory. The obtained posterior probabilities can be used to assign each individual membership to the trajectory that best 


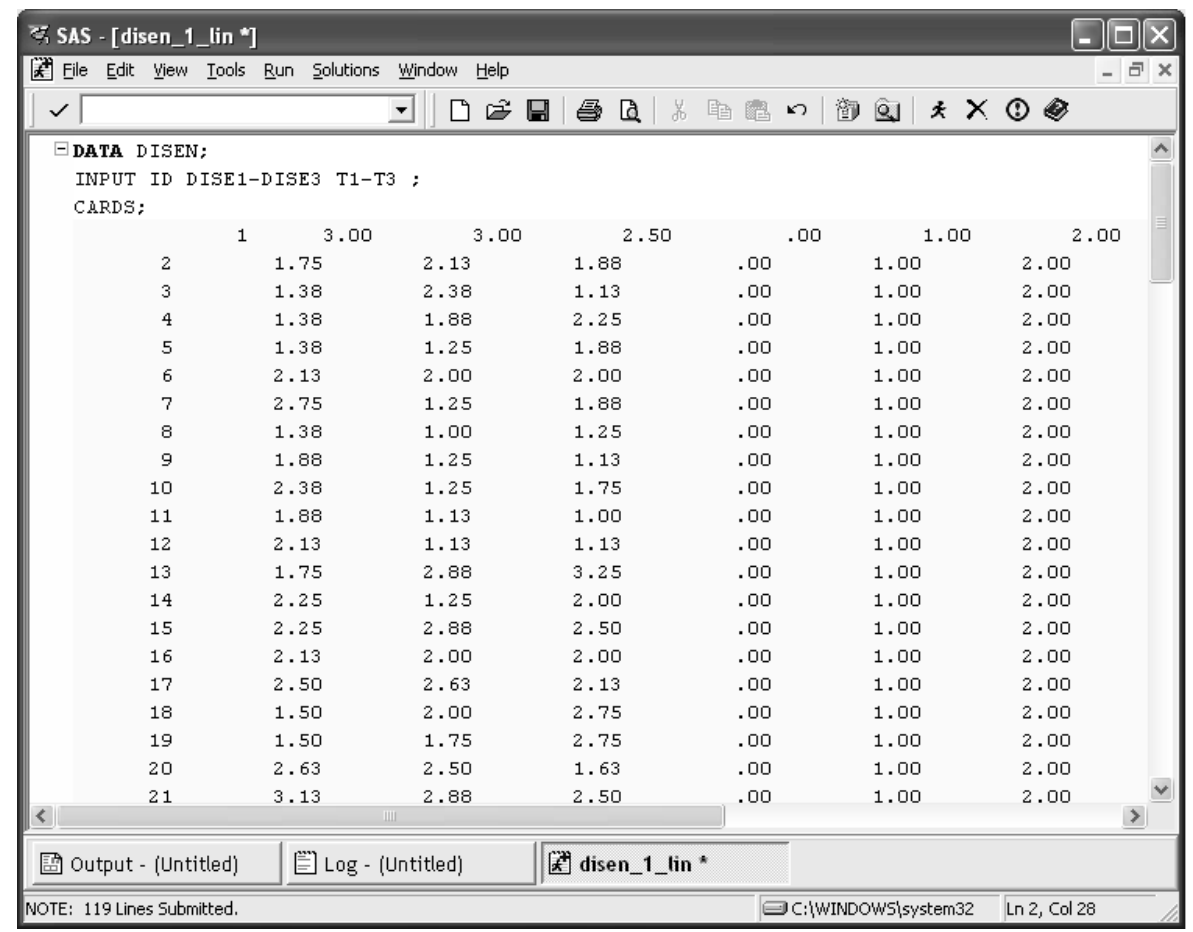

Figure 1. Syntax window with the associated data set.

matches his or her profile of change. A maximum-probability assignment rule is then used to assign each individual membership to the trajectory to which he or she holds the highest posterior membership probability. An example demonstrating the use of the maximum probability assignment rule is presented below. Table 1 displays a hypothetical data set with six individuals whose developmental course on a variable are modelled by three trajectories, thus resulting in three posterior probability values for each individual. Using the maximum probability assignment rule, participants 1 and 5 would be assigned group membership to Trajectory 3, participants 2 and 3 would be considered as members of Trajectory 2, and participants 4 and 6 would be regrouped into Trajectory 1 .

The average posterior probability of group membership is calculated for each trajectory identified in the data. The average posterior probability of group membership for a trajectory is an approximation of the internal reliability for each trajectory. This can be calculated by averaging the posterior probabilities of individuals having been assigned group membership to a trajectory using the maximum probability assignment rule. Average posterior probabilities of group membership greater than .70 to .80 are taken to indicate that the modelled trajectories group individuals with similar patterns of change and discriminate between individuals with dissimilar patterns of change. Returning to the hypothetical data set displayed in Table 1, the posterior probabilities of participants 4 and 6 with Trajectory 1 are averaged to obtain the average posterior probabilities of group membership for Trajectory $1[(0.93+1.00) \div 2=0.97]$ as are the posterior probabilities of participants 2 and 3 with Trajectory $2[(1.00+.86) \div 2=0.93]$, and the posterior probabilities of participants 1 and 5 with Trajectory 3 $[(1.00+.76) \div 2=0.88]$.

Table 1. Hypothetical data set with six participants and three trajectories

\begin{tabular}{cccc}
\hline & \multicolumn{3}{c}{ Posterior Probability } \\
\hline Participants & Trajectory 1 & Trajectory 2 & Trajectory 3 \\
\hline 1 & .10 & .10 & .80 \\
2 & .00 & $\mathbf{1 . 0 0}$ & .00 \\
3 & .04 & .86 & .10 \\
4 & .93 & .02 & .05 \\
5 & .20 & .03 & .76 \\
6 & $\mathbf{1 . 0 0}$ & .00 & .00 \\
\hline
\end{tabular}




\section{Performing LCGM using SAS}

For illustration purposes, a data set obtained with permission from Louvet, Gaudreau, Menaut, Genty, and Deneuve (2007) will be used to demonstrate how to perform the LCGM analyses (see Appendix A). This data set, labelled DISEN, includes a measure of disengagement coping at three time points for 107 participants. Typically, a data set of at least 300 to 500 cases is preferable for running LCGM, although the analysis can be applied to data sets of at least 100 cases (Nagin, 2005). It should be noted that performing LCGM with smaller sample sizes limits the power of the analysis as well as the number of identifiable trajectories (Nagin, 2005). In such instances, as in the example presented in this tutorial, the researcher may adopt a more liberal significance criterion (e.g., $p<.10$; Tabachnick \& Fidell, 2007) which is then applied consistently throughout the analysis.

In order to perform the analysis using SAS, the user has to install the PROC TRAJ application (Jones et al., 2001) which is freely available at the following website: http://www.andrew.cmu.edu/user/bjones/. Complete instructions for downloading and installing the PROC TRAJ application are available on the website (Jones, 2005).

Before running the analysis using the PROC TRAJ application in SAS, the data set to be analyzed will need to be imported from the computer program on which it was prepared (e.g., Excel). The variables from the data set should be labelled before being imported into SAS. As seen in Figure 1, the first column in our data set is labelled "ID" and identifies each case in the data set. Each row should contain the data of one individual, or case. The next columns are the dependent variable scores. In this data set, there are three measures of disengagement coping, one at each time point. Thus, in this example, the second column is disengagement coping at Time 1, the third column is disengagement coping at Time 2, and the fourth column is disengagement coping at Time 3. The next columns in the data set are variables representing the time points at which the dependent variable was measured; in this example, columns 5, 6, and 7 represent Time 1, Time 2 , and Time 3 , respectively. In this data set, each measurement point is separated by the same amount of time; therefore, Time 1 is coded as " 0 ", Time 2 is coded as " 1 ", and Time 3 is coded as " 2 ". However, LCGM can be performed even when the measurement points are separated by different time intervals that are relevant to the data analysis (e.g., Time 1 = baseline, Time $2=1$ month, Time $3=6$ months). In these instances, the user can code the time variable to represent the age or time of each measurement point (e.g., 5, 7, 13 [years old] or 1, 6, 18 [months since baseline]). The specific age/time can also vary across individuals to account for the fact that it is often impossible to measure each case at the exact same time. For this reason, the time variable is entered individually for each case in the database. Also, it is important to note that SAS uses an imputation procedure to assign values for missing data which may not be suitable for every data set (McKnight, McKnight, Sidani, \& Figueredo, 2007). Given this, it is recommended that any missing data be treated

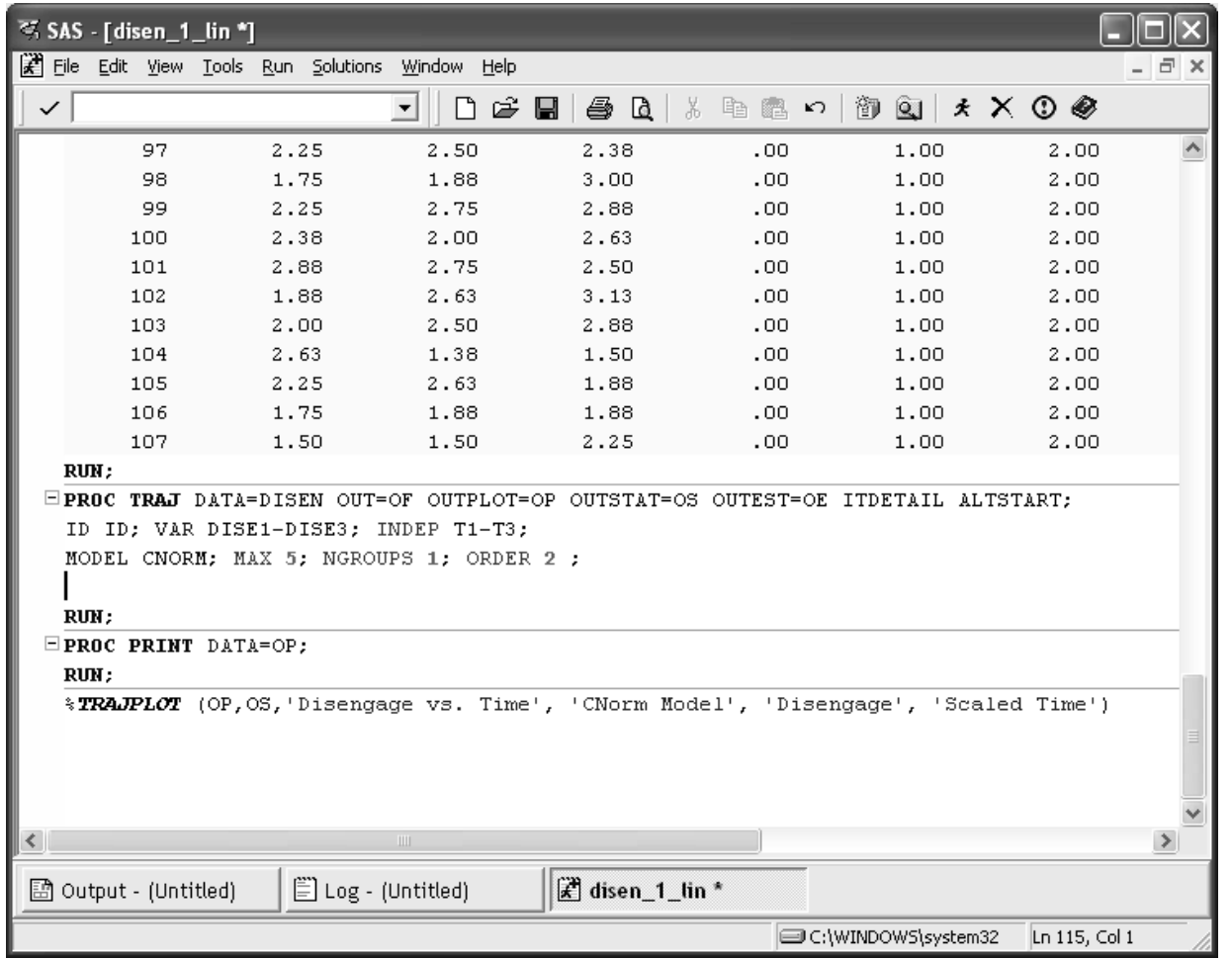

Figure 2. Syntax for the analysis of a model with one quadratic trajectory. 


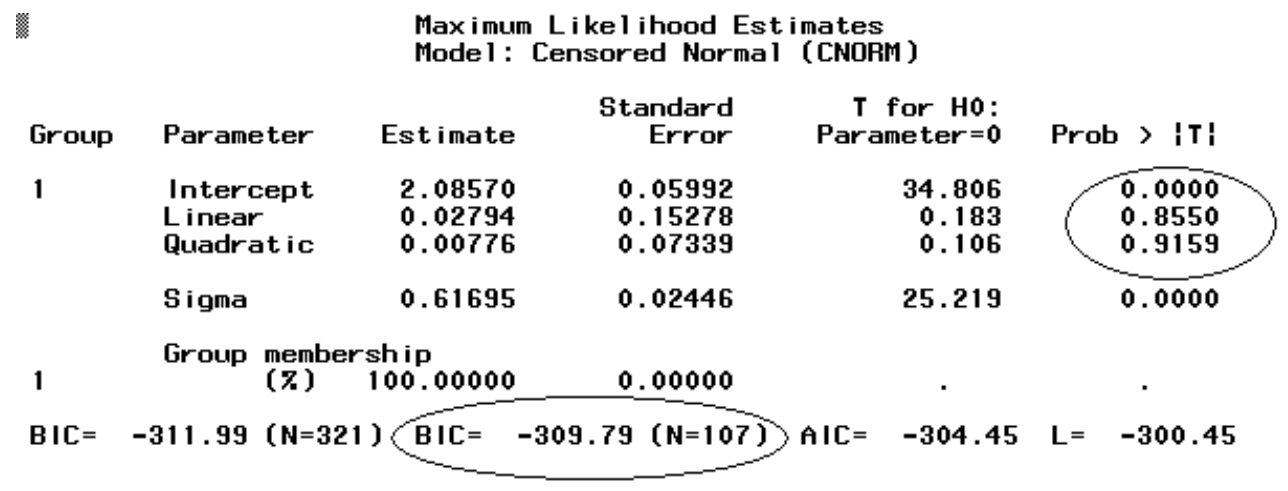

Figure 3. Output from the analysis of a model with one quadratic trajectory.

prior to importing the data set into the PROC TRAJ application.

To import the data into SAS, four steps must be followed. First, the user selects Import Data from the File drop-down menu. A window will open where the location of the data on the computer is entered. Second, the user clicks on the syntax window and enters the following three lines of syntax (please note, the colour of the text will change automatically as it is entered):
The syntax "PROC PRINT DATA=OP" provides a table of the predicted value of the dependent variable at each measurement point for each trajectory. These values are used to create a figure depicting the shape of each trajectory. SAS provides a figure with low resolution that may need to be recreated using an alternative statistical program such as SPSS if the graphs are to be used in publication or modified in any way. Figure 2 illustrates the syntax window at this point.

To run the analysis, the user clicks the icon of the person DATA $<$ NAME OF DATABASE $>$; INPUT ID $<$ DEPENDENT VARIABLES $><$ INDEPENDENT VARIABLES $>$; CARDS;

Third, once the syntax has been entered, the data set can be copied and pasted into the syntax window from a program like Excel. Finally, on the next line after the data set, the syntax "RUN;" is entered. Figure 1 provides an example of the syntax window at this point.

After adding the data set to the syntax window, the user enters the syntax to run the analysis. In this case, given that there are three time points, the first model tests the quadratic parameters for one trajectory (defined in the syntax as NGROUPS 1) using the following syntax. For a more complete description of each of the syntax items, please refer to Appendix B.

PROC TRAJ DATA $=<$ DATABASE $>$ OUT $=$ OF OUTPLOT $=$ OP OUTSTAT $=$ OS

OUTEST=OE ITDETAIL ALTSTART;

ID ID; VAR $<$ DEPENDENT VARIABLES $>$; INDEP $<$ INDEPENDENT

VARIABLES $>$;

MODEL CNORM; MAX <MAX SCORE OF DEP. VAR.>; NGROUPS 1; ORDER 2;

RUN ;

PROC PRINT DATA=OP;

RUN ; the bar at the bottom of the screen. The output for this one quadratic trajectory model is displayed in Figure 3. In each output, statistics are provided for each estimated parameter including the intercept $\left(\beta_{0}\right)$, the linear parameter $\left(\beta_{1}\right)$, and the quadratic parameter $\left(\beta_{2}\right)$ for each trajectory. The intercept $\left(\beta_{0}\right)$ corresponds to the value of the dependent variable when the value of the independent variable is equal to zero. A $t$-test for the intercept provided in the output indicates whether the value of the dependent variable significantly differs from zero when the independent variable is equal to zero. The linear slope parameter estimate $\left(\beta_{1}\right)$ represents the amount of increase/decrease on the dependent variable for each unit of increase on the independent variable (e.g., Time 1 to Time 2). The quadratic slope parameter estimate $\left(\beta_{2}\right)$ represents the amount of increase/decrease on the dependent variable for each unit of increase on the squared independent variable. The amount of variance in the data accounted for by the model and its significance is given by Sigma. The above information is 
read from the Output for each model. The Group column labels the number of trajectories tested and the Parameter column labels the estimated parameters. The test statistic, standard error, and significance for each parameter are displayed in the Estimate column, the Standard Error column, the Prob $>|T|$ column, respectively. The $T$ for $H_{0}$ : Parameter $=$ 0 column provides a value for the test of the null hypothesis that determines whether the parameter is significant or not. The value of the $t$-test has to be higher than $1.96(p<.05)$ or $2.58(p<.01)$. Two BIC values are also provided in each output and the second BIC value is interpreted as the index of fit for the model. In this analysis modelled with one quadratic trajectory, the results can be summarized as follows: $\beta_{0}=2.09, p=.000 ; \beta_{1}=.028, p=0.86$; and $\beta_{2}=.01, p=$ .92 ; Sigma $=.62, p=.000 ; \mathrm{BIC}=-309.79$ (see Figure 3 ). The user can also scroll down in this window to view the predicted values of the dependent variable at different values of the independent variable for each point on the graph.

As a general rule, for data sets with three time points, a single quadratic trajectory model is tested first. If the quadratic component of this model is not significant, the model for one linear trajectory is run to determine the BIC value for this model. If the quadratic component of the model for one trajectory is significant, the analysis for the quadratic model for two trajectories is performed. Following these analyses, the BIC value of the appropriate twotrajectory model will be compared to the BIC value of the appropriate one-trajectory model. This process is repeated with an increasing number of trajectories until the model of best fit is obtained, as determined by comparing the BIC values.

Coming back to our example, since the quadratic component of the one trajectory model was not significant, the model for one linear trajectory is run by changing the order of the model from $\mathbf{2}$ (for quadratic) to $\mathbf{1}$ (for linear) in the syntax window. The rest of the syntax remains the same. After running this analysis, a new graph and a new output with the required BIC value is also generated.

To run the analysis for the quadratic model for two trajectories the user changes the syntax from "NGROUPS 1 " to "NGROUPS 2" to estimate two trajectories. The syntax for the order is also changed to "ORDER 2 2". When using the ORDER syntax, the first number represents the first trajectory and the second number represents the second trajectory. Further, a " 2 " indicates the trajectory should be modelled on a quadratic trend whereas a " 1 " indicates a linear trend. This analysis can now be run. If neither of the quadratic components of the two-trajectory model is significant, the linear model can be run (syntax "ORDER 1 $\left.1^{\prime \prime}\right)$. Likewise, if only one component is significant, a model with one quadratic component and one linear component can be run (syntax “ORDER 12 " or “ORDER 2 1").

In our example, given that both quadratic components of the two-trajectory model are significant, the BIC value obtained from this analysis is compared to the BIC value from the previous analysis to test for improvement of fit. Using this data set as an example, the model with two quadratic trajectories (BIC $=-289.05$ ) is compared to the model with one linear trajectory $(\mathrm{BIC}=-307.79)$ using the

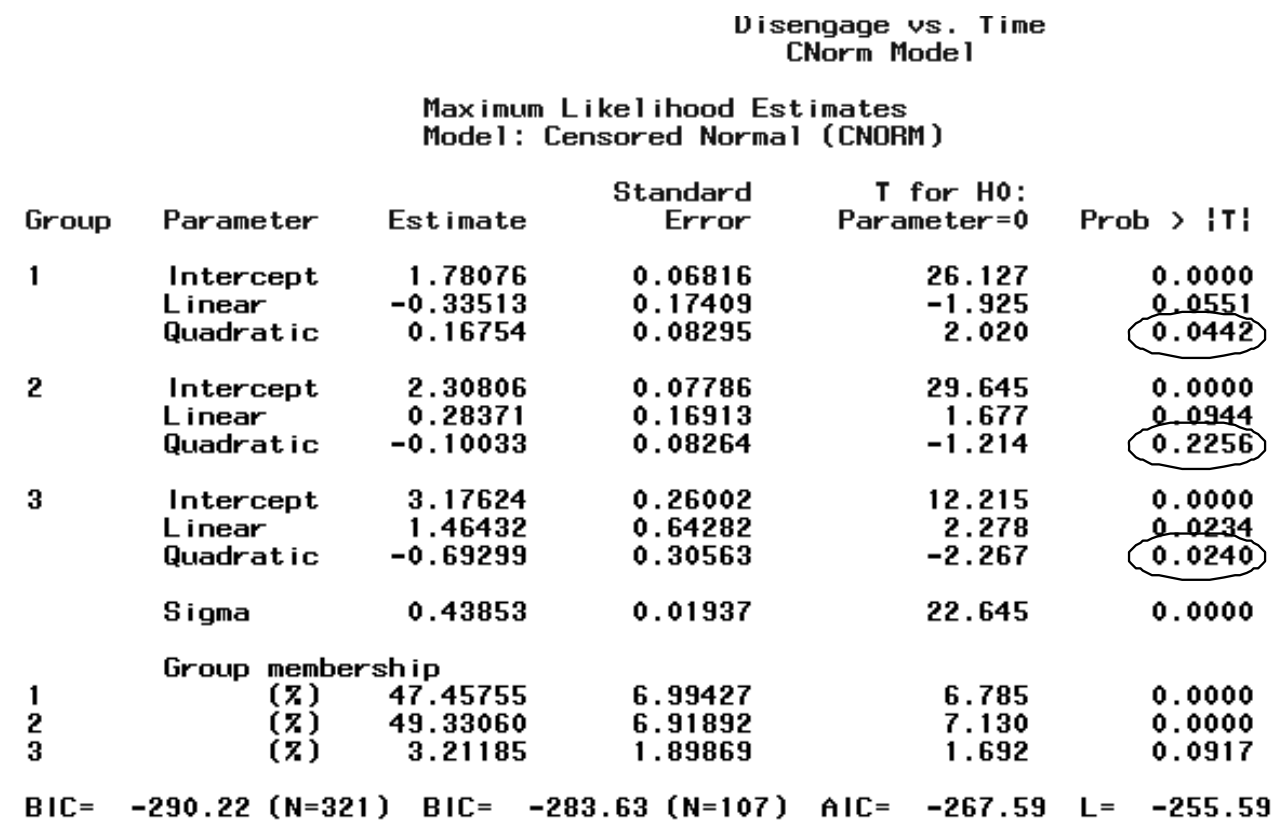

Figure 4. Output from the analysis of a model with three quadratic trajectories. 


\begin{tabular}{|c|c|c|c|c|c|c|}
\hline \multirow[b]{2}{*}{ Group } & \multicolumn{5}{|c|}{$\begin{array}{l}\text { Maximum Likelihood Estimates } \\
\text { Model: Censored Normal (CNORM) }\end{array}$} & \multirow[b]{2}{*}{ Prob > |T| } \\
\hline & \multicolumn{2}{|c|}{ Parameter } & Estimate & $\begin{array}{l}\text { Standard } \\
\text { Error }\end{array}$ & $\begin{array}{l}\text { T for } \mathrm{H} 0: \\
\text { Parameter }=0\end{array}$ & \\
\hline 1 & \multicolumn{2}{|c|}{$\begin{array}{l}\text { Intercept } \\
\text { Linear } \\
\text { Quadrat ic }\end{array}$} & $\begin{array}{r}1.77588 \\
-0.31826 \\
0.15650\end{array}$ & $\begin{array}{l}0.06911 \\
0.17634 \\
0.08398\end{array}$ & $\begin{array}{r}25.696 \\
-1.805 \\
1.864\end{array}$ & $\begin{array}{r}0.0000 \\
0.0721 \\
0.0633 \\
\end{array}$ \\
\hline 2 & \multicolumn{2}{|c|}{$\begin{array}{l}\text { Intercept } \\
\text { Linear }\end{array}$} & $\begin{array}{l}2.32836 \\
0.08646\end{array}$ & $\begin{array}{l}0.07798 \\
0.04698\end{array}$ & $\begin{array}{r}29.858 \\
1.840\end{array}$ & 0.0000 \\
\hline \multirow[t]{2}{*}{3} & \multicolumn{2}{|c|}{$\begin{array}{l}\text { Intercept } \\
\text { L inear } \\
\text { Quadrat ic }\end{array}$} & $\begin{array}{r}3.15938 \\
1.46784 \\
-0.69657\end{array}$ & $\begin{array}{l}0.25398 \\
0.63005 \\
0.29978\end{array}$ & $\begin{array}{r}12.440 \\
2.330 \\
-2.324\end{array}$ & $\begin{array}{r}0.0000 \\
0.0204 \\
0.0208 \\
\end{array}$ \\
\hline & \multicolumn{2}{|l|}{ Sigma } & 0.44030 & 0.01948 & 22.608 & 0.0000 \\
\hline $\begin{array}{l}1 \\
2 \\
3\end{array}$ & Group & $\begin{array}{l}\text { members } \\
(7) \\
(7) \\
(7)\end{array}$ & $\begin{array}{l}\text { ship } \\
46.45634 \\
50.19029 \\
3.35337\end{array}$ & $\begin{array}{l}7.19716 \\
7.12964 \\
1.92957\end{array}$ & $\begin{array}{l}6.455 \\
7.040 \\
1.738\end{array}$ & $\begin{array}{l}0.0000 \\
0.0000 \\
0.0832\end{array}$ \\
\hline$B I C=$ & 288.10 & & $B \mid C=$ & $.06(N=107)$ & -267.36 & -256.36 \\
\hline
\end{tabular}

Figure 5. Output from the analysis of a quadratic model for Trajectories 1 and 3 and linear model for Trajectory 2.

estimate of the log Bayes factor. The estimate of the log Bayes factor is calculated as follows:

$$
2 \times[(-289.05)-(-307.79)]=37.48
$$

Using the guidelines for interpreting the estimate of the $\log$ Bayes factor (Jones et al., 2001), these results provide very strong evidence for the model with two quadratic trajectories compared to a model with one trajectory. However, it is necessary to continue testing more complex models with an increasing number of trajectories in order to determine if the model with two trajectories provides the best fit to the data.

Next, a model with three quadratic trajectories is run by changing the syntax from "NGROUPS 2" to "NGROUPS 3" and by changing the order to read "ORDER 222 ". The output of this model, displayed in Figure 4, shows that the quadratic terms of Trajectory 1 and Trajectory 3 are significant whereas the quadratic parameter of Trajectory 2 is not significant. Given this, the user can test a model with two quadratic trajectories and one linear trajectory with the following syntax codes indicating that Trajectory 2 is linear: "NGROUPS 3; ORDER 21 2". As shown in Figure 5, the results of this analysis indicate that all modelled components of the trajectories can be considered significant as the small sample size warrants a more liberal significance criterion ( $p=0.10$; Tabachnick \& Fidell, 2007). Comparing this three-trajectory model to the two-trajectory model $2 \times[(-282.06)-(-289.05)]=13.98$ provides very strong evidence for the three-trajectory model.

The addition of trajectories continues until there is no significant improvement in model fit compared to the previously tested model. For this example, an analysis for four quadratic trajectories is run, followed by a model deleting the non-significant components of each of the four trajectories. Comparing this model with the three-trajectory model $2 \times[(-285.89)-(-282.06)]=-7.66$ reveals a decrease in fit for the four-trajectory model.

As a result, the three-trajectory model is retained as the final and most parsimonious model. The output for this final model is displayed in Figure 5 and the parameters presented in the output will be interpreted. Figure 6 displays the graph for this final model with Trajectory 1 shown on the bottom, Trajectory 2 in the middle, and Trajectory 3 on top. Trajectory 1 follows a quadratic trend in which disengagement coping decreases from Time 1 to Time 2 and returns to its initial level by Time 3 . The participants whose profiles of change are best represented by Trajectory 1 tended to report low levels of disengagement coping which decreased from Time 1 to Time 2 and then returned to the initial level by Time $3\left(\beta_{0}=1.78, p<0.001 ; \beta_{1}=-.032, p=0.07 ; \beta_{2}=0.16, p=\right.$ $0.06)$. Trajectory 2 , which follows a linear trend, represents the profile of change of the participants who reported moderate levels of disengagement at Time 1 followed by a marginally significant linear increase across time $\left(\beta_{0}=2.33, p\right.$ $\left.<0.001 ; \beta_{1}=0.09, p=0.07\right)$. Trajectory 3 follows a quadratic trend in which disengagement coping increases from Time 1 to Time 2 and then decreases by Time 3 . The participants whose profiles of change are best represented by Trajectory 3 tended to report high levels of disengagement coping $\left(\beta_{0}=\right.$ $\left.3.16, p<0.001 ; \beta_{1}=1.47, p=0.02 ; \beta_{2}=-0.70, p=0.02\right)$. 
Table 2. Upper and lower limits of the $95 \%$ confidence intervals

\begin{tabular}{cccccccccc} 
& \multicolumn{3}{c}{ Time 1 } & \multicolumn{3}{c}{ Time 2 } & \multicolumn{3}{c}{ Time 3 } \\
\hline Trajectory & Lower & Value & Upper & Lower & Value & Upper & Lower & Value & Upper \\
\hline 1 & 1.64 & 1.78 & 1.91 & 1.46 & 1.61 & 1.77 & 1.60 & 1.77 & 1.93 \\
2 & 2.18 & 2.30 & 2.48 & 2.30 & 2.47 & 2.53 & 2.37 & 2.47 & 2.64 \\
3 & 2.66 & 3.16 & 3.66 & 3.36 & 3.93 & 2.64 & 2.79 & 3.31 & 3.83 \\
\hline
\end{tabular}

After the selection of the most suitable model, the posterior probabilities (i.e., the likelihood of each case belonging to each trajectory) are calculated. This is done by running the syntax "PROC PRINT DATA=OF" which will generate a tabular output that identifies each case and the likelihood of each case belonging to each trajectory. Participants are then assigned group membership to a trajectory based on the maximum probability assignment rule. Finally, the posterior probabilities of the individuals assigned membership to a given trajectory are averaged to obtain the average posterior probability of group membership for each trajectory and can be examined to assess the reliability of the trajectory. For the final model, the average posterior probabilities for Trajectory 1, Trajectory 2, and Trajectory 3 were .88, .93, and .88, respectively. In addition, the group membership probabilities are provided in an output for each model (provided by the syntax OUT=OF), but only the group membership probabilities for the final model are interpreted in this example. Based on these probabilities, it is estimated that $47 \%$ of the sample is categorized within the first trajectory, $50 \%$ is categorized within the

start

RUN;

RUN; second trajectory and $3 \%$ of the sample is categorized within the third trajectory (see Figure 5).

\section{Recent Extensions of LCGM}

A recent extension to the PROC TRAJ application allows researchers to calculate the $95 \%$ confidence interval surrounding each trajectory to determine if the trajectories are overlapping at any of the measurement points (Jones \& Nagin, 2007). This is done by adding "CI95M" to the first line of syntax and "\%TRAJPLOTNEW" after the very last line of syntax. Below is an example of the syntax used to calculate the $95 \%$ confidence intervals surrounding each trajectory in the sample data set:
PROC TRAJ DATA=DISEN OUT=OF OUTSTAT=OS OTEST=OE ITDETAIL ALTSTART OUTPLOT=OP CI95M;

ID ID; VAR DISE1-DISE3;

INDEP T1-T3;

MODEL CNORM; MAX 5;

NGROUPS 3;

ORDER 212 ;

PROC PRINT DATA=OP;

\%TRAJPLOTNEW (OP,OS,'Disengage vs. Time', 'CNorm Model', 'Disengage', 'Scaled Time')

\section{Disengage vs. Time}

CNorm Model

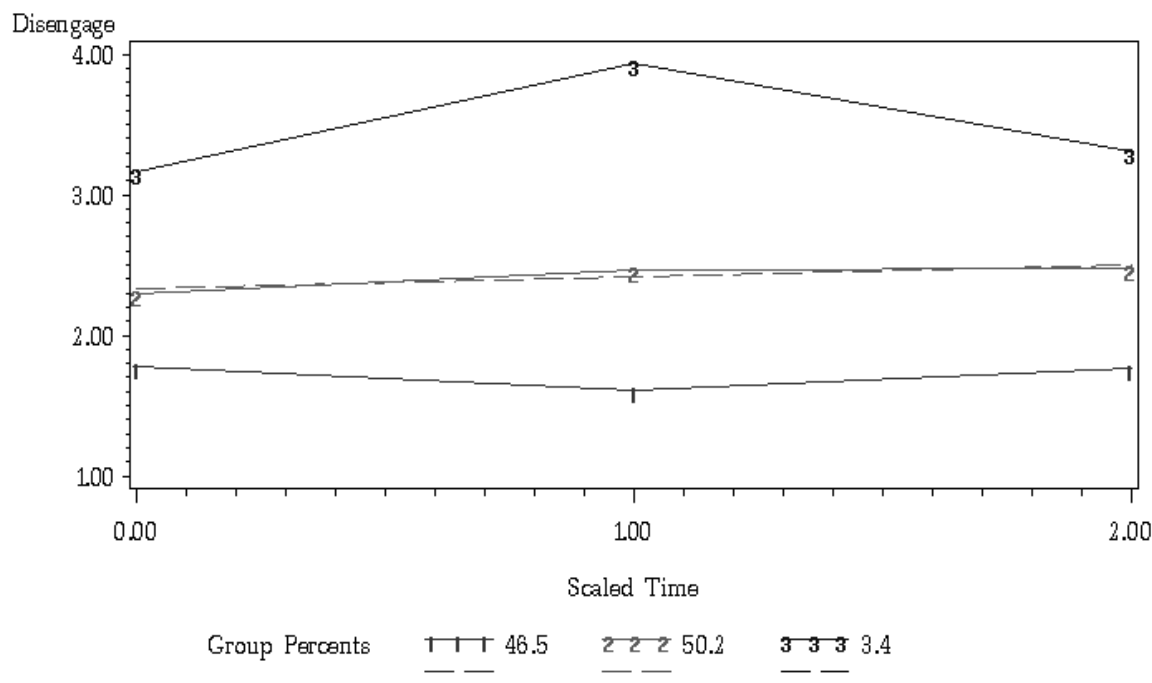

Figure 6. Graph of the final model. 
This syntax yields a new graph of the trajectories that includes the upper and lower confidence interval for each trajectory. It also provides an output with the numerical values of the confidence intervals for each trajectory at each time point, as shown in Table 2. For a more detailed description of this extension and several others, including performing LCGM with covariates, please refer to Jones and Nagin (2007) and Gaudreau, Amiot, and Vallerand (in press).

Another extension allows researchers to test whether the intercept and the slopes are significantly different across the trajectories using equality constraints, also known as invariance testing (Jones \& Nagin, 2007). The Wald test is the statistical test used to compare the intercepts $\left(\beta_{0}\right)$ as well as the linear $\left(\beta_{1}\right)$ and quadratic $\left(\beta_{2}\right)$ growth components of the trajectories to determine whether they are significantly different across trajectories. Using the sample data set, it is possible to compare if there is a significant difference between the intercepts of any two trajectories. As an example, to compare the intercepts of Trajectories 1 and 3 the following syntax is added to the line following the ORDER command: indicates that the quadratic slope of Trajectory 3 is steeper than that of Trajectory 1.

\section{Conclusion}

LCGM is a useful technique for analyzing outcome variables that change over age or time. This method provides a number of advantages over standard growth modelling procedures. First, rather than assuming the existence of a particular type or number of trajectories a priori, this method uses a formal statistical procedure to test whether the hypothesized trajectories actually emerge from the data (Nagin, 2005). As such, the method permits the discovery of unexpected yet potentially meaningful trajectories that may have otherwise been overlooked (Nagin, 2005). LCGM also bypasses a host of other challenges (e.g., over- or under-fitting the data, trajectories reflecting only random variation) associated with assignment rules sometimes used in conjunction with standard growth modelling approaches (Nagin, 2002). Although not presented in detail here, extensions of the basic LCGM model also allow the researcher to estimate the probability that an individual will belong to a particular

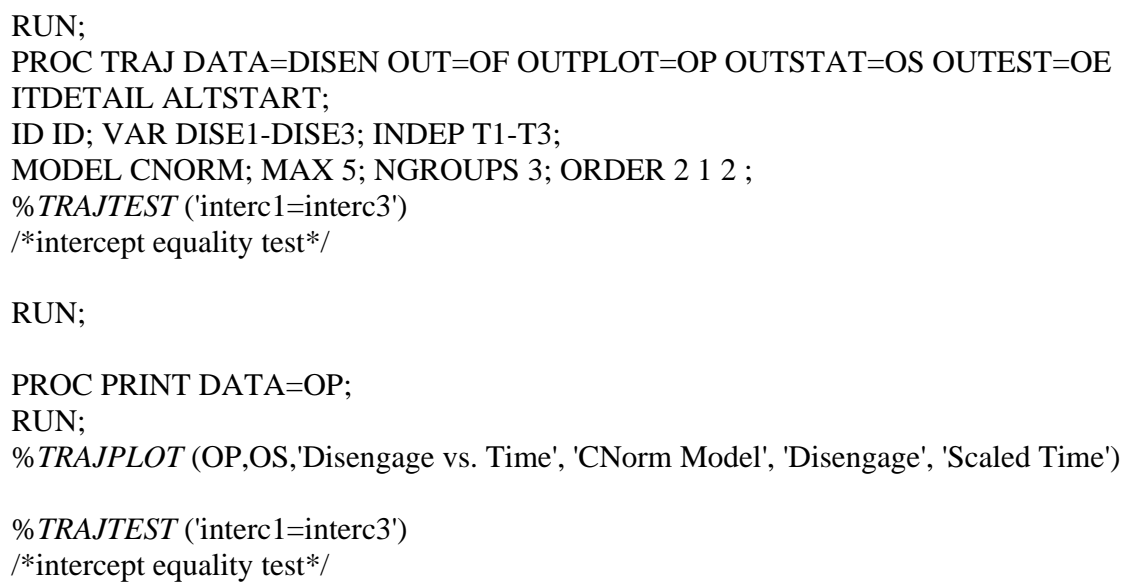

This analysis revealed that the intercepts of these two trajectories were significantly different $\left(\chi^{2}(1)=27.68, p<\right.$ .0001), thus indicating that Trajectory 3 had a greater disengagement score at the time point on which the independent variable was centered (i.e., Time 1 was coded as 0 ; see Figure 1) .To compare the slopes of Trajectories 1 and 3 , the "\%TRAJTEST" syntax is changed to read:

\%TRAJTEST('linear1=linear3, quadra3=quadra1')

/*linear\&quadratic equality test*/

The rest of the syntax remains the same and this analysis reveals that the slopes of these two quadratic trajectories are also significantly different, $\chi^{2}(2)=7.64 p<.05$, which trajectory based on their score on a covariate. Other extensions allow the researcher to obtain estimates of whether a turning point event (such as an intervention or important life transition) can alter a developmental trajectory (see Nagin, 2005; Jones \& Nagin, 2007). In addition, LCGM serves as a steppingstone to growth mixture modelling analyses in which the precise number and shape of each trajectory must be known a priori in order for the researcher to impute the requisite start values for the model to converge in software packages such as Mplus (Jung \& Wickrama, 2008). Finally, the method lends itself well to the presentation of results in graphical and tabular format, which can facilitate the dissemination of the findings to wide-ranging audiences (Nagin, 2005).

Notwithstanding the numerous advantages of LCGM, one limitation concerns the number of assessments needed to run the analysis. As with all growth models, a minimum of three time points is required for proper estimation and four or five time points are preferable in order to estimate more complex models involving trajectories following cubic or quadratic trends (Curran \& Muthén, 1999). Given the need for numerous repeated assessments, greater attrition rates are expected (Raudenbush, 2001). Attrition can weaken statistical precision and potentially introduce bias if the data 
are not missing at random (MAR) or missing completely at random (MCAR; McKnight et al., 2007).

In sum, the aim of this tutorial was to introduce readers to LCGM and to provide a concrete example of how the analysis can be performed using the SAS software package and accompanying PROC TRAJ application. With the aforementioned advantages and limitations in mind, readers are encouraged to consider LCGM as an alternative to raw and residualized change scores as well as to standard growth approaches whenever multiple and somewhat contradictory patterns of change are part of a research question. This introduction should serve as a helpful guide to researchers and graduate students wishing to use this technique to explore multinomial patterns of change in longitudinal data.

\section{References}

Curran, P. J. \& Muthén, B. O. (1999). The application of latent curve analysis to testing developmental theories in intervention research. American Journal of Community Psychology, 27, 567-595.

Helgeson, V. S., Snyder, P., \& Seltman, H. (2004). Psychological and physical adjustment to breast cancer over 4 years: Identifying distinct trajectories of change. Health Psychology, 23, 3-15.

Gaudreau, P., Amiot, C.E., \& Vallerand, R.J. (in press). Trajectories of affective states in adolescent hockey players: Turning point and motivational antecedents. Developmental Psychology.

Jones, B. L. (2005). PROC TRAJ. Retrieved April 21, 2008, from http://www.andrew.cmu.edu/user/bjones/

Jones, B.L., Nagin, D.S., \& Roeder, K. (2001). A SAS procedure based on mixture models for estimating developmental trajectories. Sociological Methods $\mathcal{E}$ Research, 29, 374-393.

Jung, T. \& Wickrama, K. A. S. (2008). An introduction to latent class growth analysis and growth mixture modelling. Social and Personality Psychology Compass, 2, 302-317.

Llabre, M. M., Spitzer, S., Siegel S., Saab, P. G., \& Schneiderman, N. (2004). Applying latent growth curve modelling to the investigation of individual differences in cardiovascular recovery from stress. Psychosomatic Medicine, 66, 29-41.
Louvet, B., Gaudreau, P., Menaut, A., Genty, J., \& Deneuve, P. (2007). Longitudinal patterns of stability and change in coping across three competitions: A latent class growth analysis. Journal of Sport E Exercise Psychology, 29, 100-117.

Louvet, B., Gaudreau, P., Menaut, A., Genty, J., \& Deneuve, P. (2009). Revisiting the changing and stable properties of coping utilization using latent class growth analysis: A longitudinal investigation with soccer referees. Psychology of Sport and Exercise, 10, 124-135.

Maughan, B. (2005). Developmental trajectory modelling: A view from developmental psychology. The ANNALS of the American Academy of Political and Social Science, 602, 118-130.

McKnight, P. E., McKnight, K. M., Sidani, S., \& Figueredo, A. J. (2007). Missing data: A gentle introduction. New York: Guilford Press.

Nagin, D. S. (1999). Analyzing developmental trajectories: A semi-parametric, group-based approach. Psychological Methods, 4, 139-157.

Nagin, D. S. (2002). Overview of a semi-parametric, groupbased approach for analyzing trajectories of development. Proceedings of Statistics Canada Symposium 2002: Modelling Survey Data for Social and Economic Research.

Nagin, D. S. (2005). Group-based modelling of development. Cambridge, MA: Harvard University Press.

Raudenbush, S. W. (2001). Comparing-personal trajectories and drawing causal inferences from longitudinal data. Annual Review of Psychology, 52, 501-25.

Roberts, B. W., Walton, K. E., \& Viechtbauer, W. (2006). Patterns of mean-level change in personality traits across the life course: A meta-analysis of longitudinal studies. Psychological Bulletin, 132, 1-25.

Sampson, R. J. \& Laub, J. H. (2005). A life-course view of the development of crime. The ANNALS of the American Academy of Political and Social Science, 602, 12-45.

Singer, J. D. \& Willet, J. B. (2003). Applied longitudinal data analysis: Modeling change and event occurrence. New York: Oxford University Press.

Tabachnick, B. G. \& Fidell, L. S. (2007). Using Multivariate Statistics ( $5^{\text {th }}$ ed.). New York: Pearson, Allyn, and Bacon.

Manuscript received October $2^{\text {nd }}, 2008$

Manuscript accepted March 11th, 2009.

(Apendices follow) 


\section{Appendix A : Sample data set}

\begin{tabular}{|c|c|c|c|}
\hline Participant & $\begin{array}{c}\text { Disengagement coping } \\
\text { (Time } 1)\end{array}$ & $\begin{array}{c}\text { Disengagement coping } \\
\text { (Time } 2 \text { ) }\end{array}$ & $\begin{array}{c}\text { Disengagement coping } \\
\text { (Time 3) }\end{array}$ \\
\hline 1 & 3.00 & 3.00 & 2.50 \\
\hline 2 & 1.75 & 2.13 & 1.88 \\
\hline 3 & 1.38 & 2.38 & 1.13 \\
\hline 4 & 1.38 & 1.88 & 2.25 \\
\hline 5 & 1.38 & 1.25 & 1.88 \\
\hline 6 & 2.13 & 2.00 & 2.00 \\
\hline 7 & 2.75 & 1.25 & 1.88 \\
\hline 8 & 1.38 & 1.00 & 1.25 \\
\hline 9 & 1.88 & 1.25 & 1.13 \\
\hline 10 & 2.38 & 1.25 & 1.75 \\
\hline 11 & 1.88 & 1.13 & 1.00 \\
\hline 12 & 2.13 & 1.13 & 1.13 \\
\hline 13 & 1.75 & 2.88 & 3.25 \\
\hline 14 & 2.25 & 1.25 & 2.00 \\
\hline 15 & 2.25 & 2.88 & 2.50 \\
\hline 16 & 2.13 & 2.00 & 2.00 \\
\hline 17 & 2.50 & 2.63 & 2.13 \\
\hline 18 & 1.50 & 2.00 & 2.75 \\
\hline 19 & 1.50 & 1.75 & 2.75 \\
\hline 20 & 2.63 & 2.50 & 1.63 \\
\hline 21 & 3.13 & 2.88 & 2.50 \\
\hline 22 & 1.25 & 1.75 & 1.13 \\
\hline 23 & 1.63 & 1.25 & 1.00 \\
\hline 24 & 2.13 & 2.50 & 2.00 \\
\hline 25 & 1.63 & 2.00 & 2.00 \\
\hline 26 & 1.88 & 3.13 & 2.00 \\
\hline 27 & 1.88 & 1.63 & 1.63 \\
\hline 28 & 2.00 & 2.50 & 3.25 \\
\hline 29 & 1.50 & 1.13 & 1.50 \\
\hline 30 & 1.75 & 2.38 & 2.25 \\
\hline 31 & 2.25 & 1.63 & 1.50 \\
\hline 32 & 2.00 & 1.63 & 1.25 \\
\hline 33 & 1.63 & 1.25 & 3.00 \\
\hline 34 & 2.13 & 1.50 & 2.50 \\
\hline 35 & 2.00 & 3.25 & 2.75 \\
\hline 36 & 1.38 & 2.63 & 1.75 \\
\hline 37 & 2.13 & 1.25 & 2.00 \\
\hline 38 & 2.63 & 2.38 & 2.25 \\
\hline 39 & 2.13 & 2.75 & 1.50 \\
\hline 40 & 2.63 & 2.00 & 2.50 \\
\hline 41 & 2.00 & 2.63 & 2.25 \\
\hline 42 & 1.88 & 1.13 & 1.25 \\
\hline 43 & 2.38 & 2.75 & 2.13 \\
\hline 44 & 1.50 & 1.50 & 1.88 \\
\hline 45 & 1.63 & 1.38 & 2.63 \\
\hline 46 & 2.63 & 2.13 & 2.38 \\
\hline 47 & 1.75 & 1.13 & 1.38 \\
\hline 48 & 1.88 & 2.25 & 1.88 \\
\hline 49 & 2.38 & 4.25 & 4.63 \\
\hline 50 & 1.50 & 2.13 & 1.88 \\
\hline 51 & 1.63 & 1.38 & 1.63 \\
\hline 52 & 3.25 & 2.13 & 2.38 \\
\hline 53 & 2.75 & 2.25 & 2.13 \\
\hline 54 & 2.00 & 3.00 & 2.63 \\
\hline 55 & 2.13 & 1.38 & 2.38 \\
\hline 56 & 2.13 & 2.63 & 2.75 \\
\hline
\end{tabular}




\section{Appendix A (continued)}

\begin{tabular}{|c|c|c|c|}
\hline Participant & $\begin{array}{c}\text { Disengagement coping } \\
\text { (Time 1) }\end{array}$ & $\begin{array}{l}\text { Disengagement coping } \\
\text { (Time 2) }\end{array}$ & $\begin{array}{l}\text { Disengagement coping } \\
\text { (Time 3) }\end{array}$ \\
\hline 57 & 1.88 & 1.75 & 2.25 \\
\hline 58 & 1.63 & 1.25 & 1.13 \\
\hline 59 & 1.50 & 1.63 & 1.25 \\
\hline 60 & 1.88 & 2.13 & 2.00 \\
\hline 61 & 1.50 & 1.50 & 1.88 \\
\hline 62 & 2.13 & 1.50 & 1.75 \\
\hline 63 & 2.75 & 3.63 & 2.75 \\
\hline 64 & 1.38 & 1.13 & 2.38 \\
\hline 65 & 2.25 & 2.50 & 2.50 \\
\hline 66 & 2.13 & 1.88 & 1.75 \\
\hline 67 & 1.38 & 2.00 & 2.25 \\
\hline 68 & 1.88 & 2.00 & 2.25 \\
\hline 69 & 1.75 & 2.00 & 1.38 \\
\hline 70 & 3.50 & 4.50 & 2.63 \\
\hline 71 & 3.25 & 2.50 & 2.38 \\
\hline 72 & 1.38 & 1.25 & 1.25 \\
\hline 73 & 2.88 & 2.88 & 2.88 \\
\hline 74 & 2.25 & 2.75 & 2.25 \\
\hline 75 & 2.38 & 2.38 & 2.25 \\
\hline 76 & 3.25 & 2.13 & 2.38 \\
\hline 77 & 2.13 & 2.13 & 2.50 \\
\hline 78 & 1.75 & 2.00 & 2.63 \\
\hline 79 & 2.38 & 3.13 & 3.00 \\
\hline 80 & 1.25 & 2.13 & 1.75 \\
\hline 81 & 2.63 & 2.13 & 3.00 \\
\hline 82 & 1.25 & 2.00 & 1.50 \\
\hline 83 & 2.13 & 2.00 & 1.75 \\
\hline 84 & 1.75 & 2.38 & 3.00 \\
\hline 85 & 2.00 & 3.00 & 2.75 \\
\hline 86 & 2.25 & 2.75 & 2.63 \\
\hline 87 & 2.88 & 1.75 & 2.13 \\
\hline 88 & 3.88 & 3.25 & 3.00 \\
\hline 89 & 3.13 & 2.75 & 3.00 \\
\hline 90 & 2.25 & 2.00 & 2.25 \\
\hline 91 & 2.38 & 3.25 & 2.00 \\
\hline 92 & 2.25 & 2.13 & 3.13 \\
\hline 93 & 2.38 & 2.00 & 2.63 \\
\hline 94 & 1.38 & 1.88 & 2.38 \\
\hline 95 & 1.63 & 1.00 & 1.75 \\
\hline 96 & 2.00 & 2.63 & 2.00 \\
\hline 97 & 2.25 & 2.50 & 2.38 \\
\hline 98 & 1.75 & 1.88 & 3.00 \\
\hline 99 & 2.25 & 2.75 & 2.88 \\
\hline 100 & 2.38 & 2.00 & 2.63 \\
\hline 101 & 2.88 & 2.75 & 2.50 \\
\hline 102 & 1.88 & 2.63 & 3.13 \\
\hline 103 & 2.00 & 2.50 & 2.88 \\
\hline 104 & 2.63 & 1.38 & 1.50 \\
\hline 105 & 2.25 & 2.63 & 1.88 \\
\hline 106 & 1.75 & 1.88 & 1.88 \\
\hline 107 & 1.50 & 1.50 & 2.25 \\
\hline
\end{tabular}




\section{Appendix B : Documentation of Syntax}

(reprinted with permission from Jones, 2005)

INPUT NAME:

Specify DATA= data for analysis, e.g. DATA=ONE.

OUTPUT NAMES::

OUT= Group assignments and membership probabilities, e.g. OUT=OF.

OUTSTAT $=$ Parameter estimates used by TRAJPLOT macro, e.g. OUTSTAT=OS.

OUTPLOT $=$ Trajectory plot data, e.g. OUTPLOT=OP.

OUTEST= Parameter and covariance matrix estimates, e.g. OUTEST=OE.

ADDITIONAL OPTIONS:

ITDETAIL displays minimization iterations for monitoring model fitting progress.

ALTSTART provides a second default start value strategy.

ID; Variables (typically containing information to identify observations) to place in the output (OUT=) data set, e.g. ID IDNO;

VAR; Dependent variables, measured at different times or ages (for example, hyperactivity score measured at age $t$,)

e.g. VAR V1-V8;

INDEP; Independent variables (e.g. age, time) when the dependent (VAR) variables were measured, e.g. INDEP T1-T8;

MODEL; Dependent variable distribution (CNORM, ZIP, LOGIT) e.g. MODEL CNORM;

MIN; (CNORM) Minimum for censoring, e.g. MIN 1; If omitted, MIN defaults to zero.

MAX; (CNORM) Maximum for censoring, e.g. MAX 6; If omitted, MAX defaults to +infinity.

ORDER; Polynomial ( 0 =intercept, 1=linear, 2=quadratic, 3=cubic) for each group, e.g. ORDER 222 0; If omitted, cubics are used by default. 